\title{
Infrared image edge detection applied research based on improved ant colony algorithm
}

\author{
B.M. Qiao ${ }^{1, a}$, S. Li ${ }^{1, b}$ and Y. Xiao ${ }^{1, c}$ \\ ${ }^{1}$ Science and Technology of Xi'an University, Shannxi Xian, 710054, China \\ a qiaobm@xust.edu.cn, 'beerqingting1219@163.com, 'xiaoyang@xust.edu.cn
}

Keywords: Histogram equalization method, Infrared image, Ant colony algorithm

\begin{abstract}
The paper based on the analysis of ant colony algorithm and any improved algorithm, We change the initial position of the ant placed from randomly into placed near the edge points and define bounded domain node gradient values as the heuristic information,and also adaptive computing method is used to reach the final threshold.Meanwhile histogram equalization method for image preprocessing is introduced.Simulation experiment results show that this algorithm can better continuously detected edges and test results are efficient and accurate. This algorithm provides a theoretical basis for the more effective detection in infrared image edge character.
\end{abstract}

\section{Introduction}

Ant Colony Optimization (ACO) is a kind of distributed intelligence simulation algorithm, the basic idea is to imitate ants which rely on pheromones to communicate and show the social behavior[1].That is to use biological information as the basis of ants to select subsequent behavior and through the cooperation and interaction between ants to complete global optimization search process.

Nezamabadi - pour is the first successful person who realized image edge detection based on ant colony algorithm in 2005[2].Then many scholars put forward many improved algorithm, such as the improvement based on stimulating factor, combine with other swarm intelligence algorithm or merge with classic image edge detection technology.Literature [3] proposed the use of gradient image gray value change as the heuristic information of edge detection and join the movement factor change quantity, and change quantity inspire ant colony move to the edge .Literature [4] proposed to integrate the traditional Canny edge detection operator with ant colony algorithm, different pixels proportion and image angular points which Canny operator get as a priori knowledge of edge points,through the calculation of ant colony algorithm to realize image edge extraction.Literature [5] put forward the idea of using genetic algorithm to improve the ant colony algorithm, and variation factors which can be adjusted with algorithms process were introduced in the algorithm , and so on.

In this paper, the histogram equalization method is applied to preprocess images to enhance the contrast of images, then a kind of improved ant colony algorithm is proposed according to the characteristics of infrared image and applied it to the infrared image edge detection.

\section{ACO algorithm and its improvements}

\section{Improved ACO (Improve Ant Colony Optimization, IACO)}

Initialize improvements: ant's initial position is amended and place it from random into near edge, the specific approach is to take the image gray gradient threshold to optimize this process.

Inspired pheromone improvements: Neighborhood node gradient values will be defined as heuristic information, then we can get the calculating formula of heuristic information as

$$
\eta_{i, j}=\frac{1}{1+\frac{n}{\sum_{i=0}^{8}\left|I_{0}-I_{i}\right|}}
$$

Among them, $\mathrm{n}$ is the number of neighborhood nodes, $I_{i}$ is the the node $\mathrm{i}$ in adjacent domain gradient value. 
Improved termination conditions: the initial threshold value $T^{(0)}$ can select the average of all pixels pheromone matrix, then the pheromone matrix can be divided into two groups according to greater value than $T^{(0)}$ or less value than $T^{(0)}$ and new threshold value is computed as the average of two categories values .

IACO algorithm process:

Step.1 Initialization: First place $\mathrm{K}$ ants on the image I which is composed of $\mathrm{M} \times \mathrm{N}$ pixels and each pixel is seen as an undirected graph node which ants will crawl.

Step.2 Preliminary construction: Randomly selecting one of ants from the $\mathrm{K}$ in the $\mathrm{k}$ step execution process, then make this ant move $L$ continuous step on the image .The ant move from node $(\mathrm{l}, \mathrm{m})$ to adjacent nodes $(\mathrm{i}, \mathrm{j}$ ) according to a transforming probability which is defined as follows, the state transition probability formula in the searching process is as follows:

$$
p_{l, m}^{k}(i, j)=\left\{\begin{array}{cc}
\frac{\left[\tau_{i, j}(\mathrm{t})\right]^{\alpha} \cdot\left[\eta_{i, j}\right]^{\beta}}{\sum_{l \in J_{i}^{k} \cap D}\left[\tau_{i, j}(\mathrm{t})\right]^{\alpha} \cdot\left[\eta_{i, j}\right]^{\beta}} & j \notin J_{i}^{k} \\
0 & j \in J_{i}^{k}
\end{array}\right.
$$

Among them, the ant k position is $(\mathrm{i}, \mathrm{j}), \tau_{i, j}(t)$ express the pheromone strength in vertex $\mathrm{j}$ when iteration t occurs, $\eta_{i, j}$ express the inspiration information from vertice $i$ to vertice $\mathrm{j}, \alpha, \beta$ respectively express the pheromone matrix and heuristic matrix relative importance.

Step.3 Update process: A local update for the first time: updating vertex pheromone intensity when ant move every step, updating process as follows:

$$
\tau_{i, j}(t)=(1-\rho) \cdot \tau_{i, j}(t)+\Delta \tau_{i, j}(t)
$$

$\rho(0<\rho<1)$ is pheromone volatilization coefficient, $\Delta \tau_{i, j}(t)$ represents the sum of the released information element which ants moves to $\mathrm{j}$.

A global update in the second time: update as follows after ants finish exercise

$$
\tau_{i, j}(t+1)=(1-\varphi) \cdot \tau_{i, j}(t)+\varphi \tau_{0}
$$

Among them, $\varphi$ is global pheromone volatilization factor, $\tau_{0}$ is pheromone matrix that given in advance in the initial state.

Step.4 Decision making process: we adopt the adaptive method to select the threshold value.

\section{Simulation experiment results and analysis}

Histogram equalization simulation: Fig.1 (a) and (b) are respectively expressed the original infrared image grayscale and its histogram. Here you can see that the original figure is darker and its dynamic range is small, which reflected in the histogram is that histogram gray value range is narrow and focus on the low side of grey value. Fig.1 (c) and (d) are respectively expressed the original histogram equalization result image and its corresponding histogram, now the histogram occupies the range which image grey value allowed.

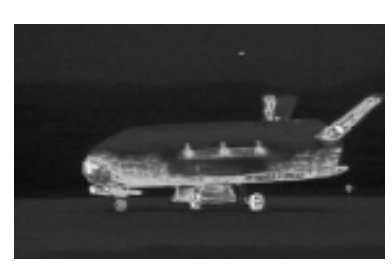

(a)

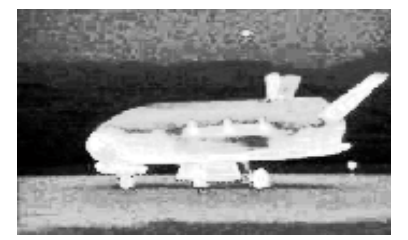

(c)

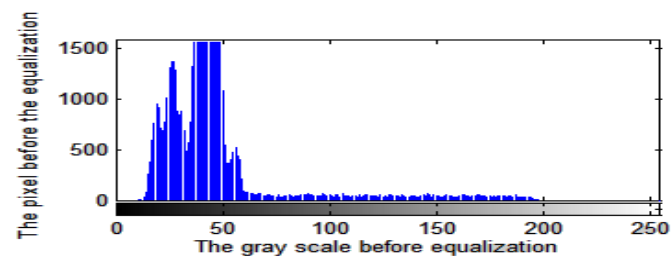

(b)

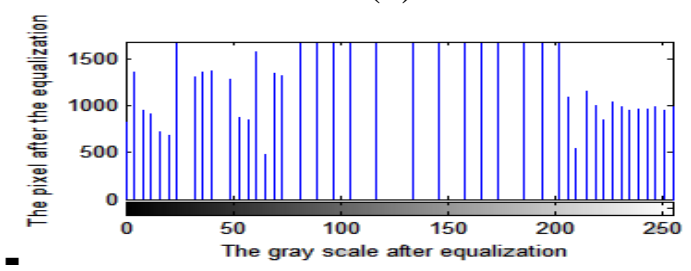

(d)

Fig.1. The contrast figure of infrared image equalization simulation analysis 
Gray image has great contrast and many details can be seen more clearly from Fig.1. The histogram equalization enhance the image gray dynamic range and also increase image contrast, thus it's good for the next edge extraction.

Image edge detection simulation analysis based on IACO algorithm

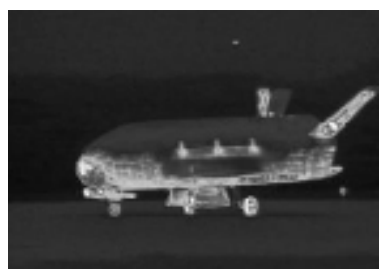

plane

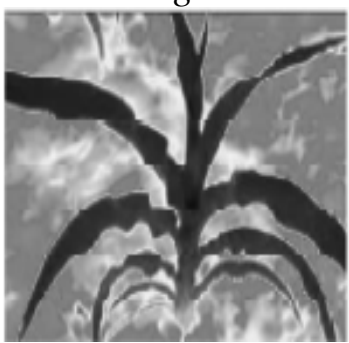

corn

Fig.2. Images to be processed

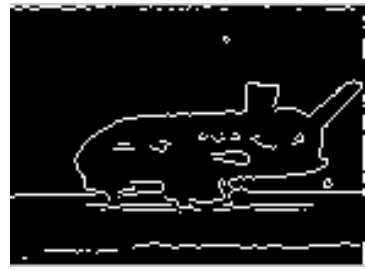

(1)Sobel algorithm

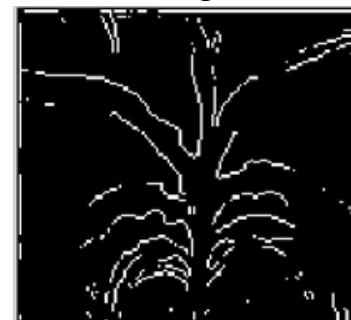

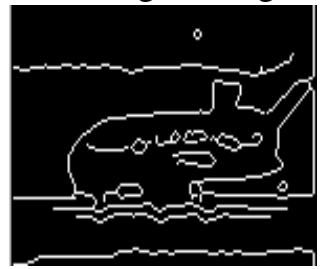

(2)Canny algorithm

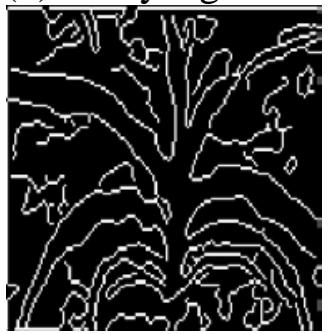

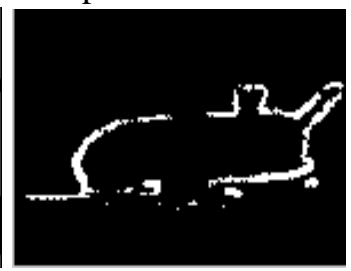

(3)ACO algorithm

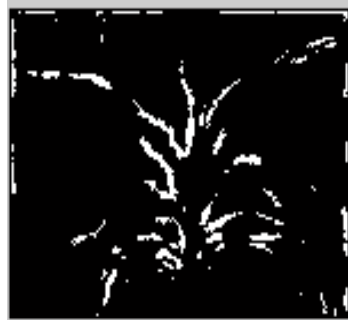

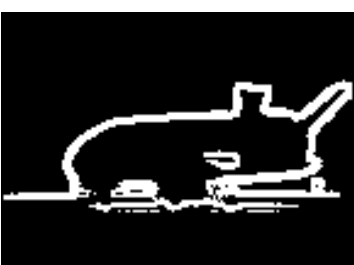

(4)IACO algorithm

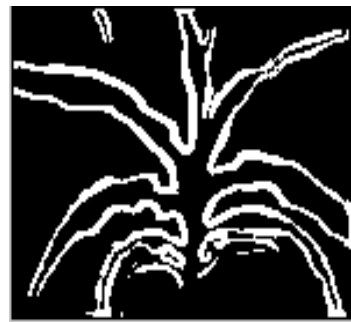

(1)Sobel algorithm (2)Canny algorithm (3)ACO algorithm (4)IACO algorithm

Fig. 3. Experiments contrast of different edge detection algorithms

Fig.3 illustrates that Sobel and Canny algorithm detect the edge with discontinuity and also regard some noise as edge points, but some the real edge points are not detected. Compared with Sobel and Canny algorithm, it detects many distortional and discontinuous edge details based on ACO algorithm. Edge can be better continuously detected by IACO algorithm and test results are efficient and accurate.

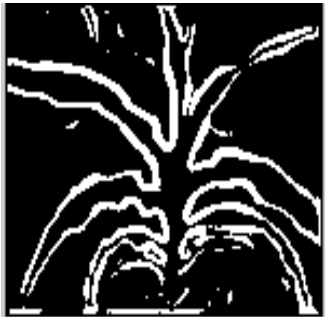

(a) $\alpha=0.6, \beta=0.1$

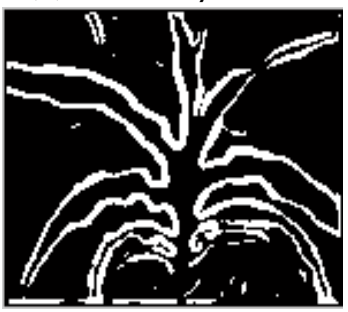

(e) $\alpha=0.8, \beta=0.1$

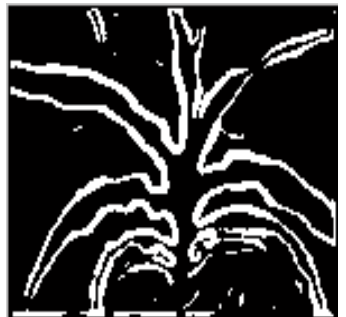

(b) $\alpha=0.8, \beta=0.1$

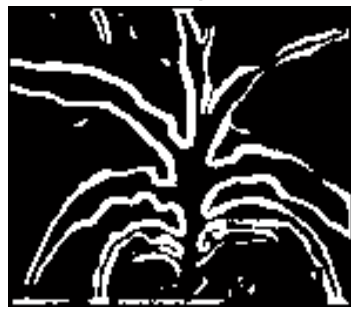

(f) $\alpha=0.8, \beta=0.2$

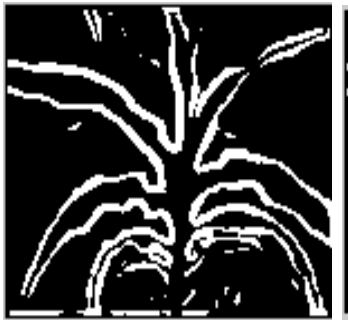

(c) $\alpha=1 \beta=0.1$

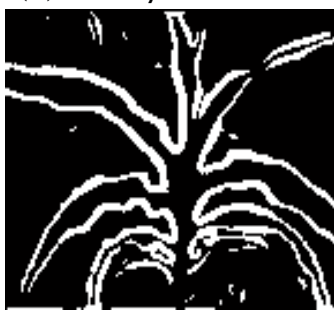

(g) $\alpha=0.8, \beta=0.5$

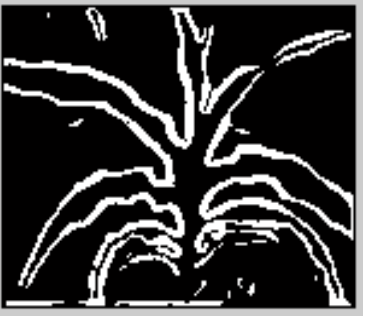

(d) $\alpha=4, \beta=2$

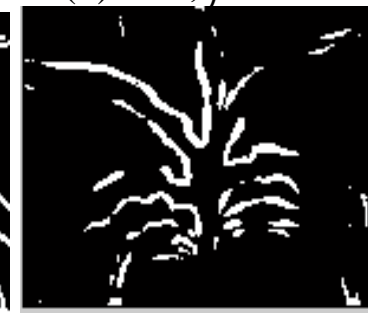

(h) $\alpha=2, \beta=4$

Fig.4. Edge detection results with different $\alpha, \beta$

The scope of heuristic information $\eta$ and the initial pheromone $\tau_{0}$ are both $[0,1][6]$, the scope[0,1] is better value of $\alpha, \beta$ from fig.4 (a) (d).Meanwhile it is better when $\alpha$ value is greater than value $\beta$ from fig.4 (e) $\sim(\mathrm{h})$. The value $\alpha=0.8$ and $\beta=0.2$ are obtained in this simulation. 
Time performance comparison between IACO algorithm and other algorithms

In order to demonstrate the effectiveness of IACO algorithm, making edge detection time performance comparison table among genetic algorithm and ACO algorithm and IACO algorithm, results are shown in table.1.

Table.1 Edge detection time performance comparison

\begin{tabular}{lccc}
\hline Image & $\mathrm{t}_{\text {genetic algorithm }}(\mathrm{s})$ & $\mathrm{t}_{\mathrm{ACO}}(\min )$ & $\mathrm{t}_{\mathrm{IACO}}(\mathrm{s})$ \\
\hline Plane & 116.19 & 7.53 & 120.47 \\
Corn & 148.31 & 9.4 & 140.68 \\
\hline
\end{tabular}

The table.1 illustrates that Genetic algorithm and IACO algorithm time-consuming are basic quite .IACO algorithm time performance is superior to ACO algorithm, that is to say that IACO algorithm has time superiority.

Edge evaluation

NP means detected edge points number, NL means 4 connected domain number in the edge image, the value of NL/NP just reflects edge linear connection degree.Algorithm performance contrast table as table. 2

Table.2 Algorithm performance contrast table

\begin{tabular}{lccccccccc}
\hline Image & \multicolumn{3}{c}{ Canny algorithm } & \multicolumn{3}{c}{ ACO algorithm } & \multicolumn{3}{c}{ IACO algorithm } \\
\cline { 2 - 10 } & NP & NL & NL/NP & NP & NL & NL/NP & NP & NL & NL/NP \\
\hline Plane & 11409 & 247 & 0.0216 & 5657 & 28 & 0.0050 & 3356 & 12 & $\mathbf{0 . 0 0 3 6}$ \\
Corn & 20840 & 858 & 0.0412 & 1667 & 117 & 0.0702 & 11440 & 53 & $\mathbf{0 . 0 0 4 6}$ \\
\hline
\end{tabular}

The table. 2 indicates that the value of NL/NP by use of the ACO algorithm and IACO algorithm are much smaller than the traditional Canny algorithm, especially IACO algorithm resulting value is the smallest.This indicates that adopting IACO algorithm to get the edge with a good linear connection degree and the best effect.

\section{Conclusion}

Regard thermal infrared image as the test object and explore IACO algorithm application in infrared image edge detection, and this paper proposes IACO algorithm for thermal infrared image edge detection algorithm. Simulation results indicate that IACO algorithm can effectively extract continuous and stable thermal infrared image edges. IACO algorithm performance is better than traditional Canny algorithm and ACO algorithm in edge evaluation, good linear connection degree and best effect can be achieved.

\section{References}

[1] Zhan Li-Li: Optimization theory and method (China mining university press, Xuzhou, 2012).

[2] Nezamabadi-pour H, Saryazdi S and Rashedi E: Edge detection using ant algorithms, J.Soft Computing, Vol. 10(7): 623-628(2006).

[3] De Wei-Gao, Tian Huang-Chen and Liu Peng: Ant colony algorithm application research in image edge detection,” J. Computer and digital engineering, Vol.5 (1) (2009), p.131.

[4] XueFu- Lu: Image edge detection based on ant colony algorithm, Chengdu: Electronic science and Technology University. Vol. 5, (2010).

[5]Huan Qing-Xie: Improved ant colony algorithm in the application of image edge detection research, Lanzhou, Lanzhou University, (2010).

[6] Xiao Qian-Zhan, He Kun, Sheng Gen-Ju, Zhang Jian and Ji Liu-Zhou: Image edge detection based on improved ant colony algorithm, Journal of Sichuan University (natural science edition). Vol. 11 (2010), p. 1265. 